\title{
Development of an integrated digital health intervention to promote engagement in and adherence to medication for opioid use disorder
}

\author{
Kirsten J. Langdon ${ }^{1,2^{*}} \mathbb{D}$, Susan Ramsey ${ }^{2,3,4}$, Caroline Scherzer ${ }^{1,5}$, Kate Carey ${ }^{6,7}$, Megan L. Ranney ${ }^{5,8}$ \\ and Josiah Rich ${ }^{3,9}$
}

\begin{abstract}
Background: Buprenorphine-naloxone is an evidence-based treatment for Opioid Use Disorder. However, despite its efficacy, nearly half of participants are unsuccessful in achieving stabilization (i.e., period of time following medication induction in which medication dose is adjusted to be effective in reducing cravings/withdrawal, minimize potential side effects, and eliminate illicit substance use). This paper presents the study design and protocol for a digital health intervention designed to promote engagement in and adherence to buprenorphine treatment, offered through an outpatient addiction treatment center, through motivational enhancement and distress tolerance skills training. Personalized feedback interventions represent a promising method to effectively motivate engagement in and adherence to buprenorphine treatment. These interventions are generally brief, individually tailored, and have the potential to be delivered via mobile platforms. Distress tolerance, a transdiagnostic vulnerability factor, has been implicated in the development and maintenance of substance use. Targeting distress tolerance may improve substance use treatment outcomes by promoting the ability to persist in goal-directed activity even when experiencing physical or emotional distress.
\end{abstract}

Methods: The study aims are to: (1) develop and refine an interactive computer- and text message-delivered personalized feedback intervention that incorporates distress tolerance skills training for persons who have elected to initiate outpatient buprenorphine treatment (iCOPE); (2) examine the feasibility, acceptability, and preliminary efficacy of iCOPE for increasing abstinence, adherence, and retention in treatment compared to a treatment as usual comparison condition; and, (3) examine potential mechanisms that may underlie the efficacy of iCOPE in improving outcomes, including motivation, distress tolerance, self-regulation, and negative affect.

Discussion: Results of this study will be used to determine whether to proceed with further testing through a largescale trial. This work has the potential to improve treatment outcomes by reducing illicit opioid use, increasing adherence/retention, and preventing future overdose and other complications of illicit opioid use.

Trial Registration NCT03842384

*Correspondence: kirsten.langdon@lifespan.org

1 Department of Psychiatry, Rhode Island Hospital, 146 West River Street,

Suite 11A, Providence, RI 02904, USA

Full list of author information is available at the end of the article

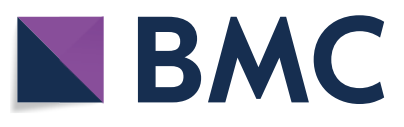

(c) The Author(s) 2020. This article is licensed under a Creative Commons Attribution 4.0 International License, which permits use, sharing, adaptation, distribution and reproduction in any medium or format, as long as you give appropriate credit to the original author(s) and the source, provide a link to the Creative Commons licence, and indicate if changes were made. The images or other third party material in this article are included in the article's Creative Commons licence, unless indicated otherwise in a credit line to the material. If material is not included in the article's Creative Commons licence and your intended use is not permitted by statutory regulation or exceeds the permitted use, you will need to obtain permission directly from the copyright holder. To view a copy of this licence, visit http://creativecommons.org/licenses/by/4.0/. The Creative Commons Public Domain Dedication waiver (http://creativecommons.org/publicdomain/zero/1.0/) applies to the data made available in this article, unless otherwise stated in a credit line to the data. 
Keywords: Opioid use disorder, Buprenorphine, Medications for opioid use disorder, Distress tolerance, Motivational enhancement, Mobile treatment

\section{Background}

The opioid overdose epidemic continues to surge across the country, resulting in a record number of fatal overdoses in 2017 [1]. With more than 2 million individuals meeting criteria for opioid use disorder (OUD) [2], there is need to expand access to evidence-based treatments. Medications for opioid use disorder (MOUD) is the current gold standard treatment for this population [3]. Of the FDA-approved MOUD, buprenorphinenaloxone (buprenorphine), a long-acting partial opioid agonist, has grown in popularity because of its more flexible administration through office-based programs [4]. Buprenorphine works by reducing cravings, alleviating withdrawal symptoms, and blocking the euphoric effects of opioids [5]. Meta-analyses highlight the effectiveness of buprenorphine in reducing adverse outcomes [6]. However, a significant proportion of patients return to illicit opioid use and/or discontinue treatment before achieving stabilization (i.e., period of time following medication induction in which medication dose is adjusted to be effective in reducing cravings/withdrawal, minimize potential side effects, and eliminate illicit substance use) $[5,7,8]$. This is notable as early lapses are related to poor treatment prognosis and retention $[8,50]$.

A negative reinforcement model of substance use posits that individuals may use opioids to mitigate aversive internal states $[8,9]$. Consistent with this perspective, negative affect has been cited as a primary precipitant of early lapse among opioid users [10]. Distress tolerance (DT), defined as the perceived or actual ability to handle uncomfortable physical or emotional states, is a transdiagnostic vulnerability factor implicated in the development and maintenance of affective symptoms/disorders and substance use [11, 12]. DT involves the ability to withstand negative reinforcement opportunities by exerting control over behavioral responding that would otherwise provide immediate relief from distress [13]. DT is inversely related to a range of drug use outcomes, including frequency and severity of use [14, 15], premature termination of treatment [16], and early lapse/relapse [17, 18]. Preliminary work suggests that addressing DT during substance use treatment may improve outcomes by promoting the ability to persist in goal directed activity even when experiencing distress and discomfort [19].

Personalized feedback interventions (PFI), such as decisional balance feedback, represent a promising method to effectively motivate engagement in and adherence to buprenorphine treatment, particularly among individuals characterized by low DT. Decisional balance feedback involves an evaluation of the advantages/disadvantages of engaging in a certain behavior (e.g., opioid use), compared to the advantages/disadvantages of an alternative behavior (e.g., abstinence), and also offers strategies for changing problematic behavior (e.g., DT skills training) $[20,21]$. Although PFIs have shown promise for reducing substance use across a variety of adult populations [21, 22], no integrated protocols exist to enhance motivation and facilitate DT skills training among individuals with OUD.

Given that negative internal states may occur in participants' natural environments in response to drug cues, stressors, or the context of withdrawal, this population may benefit from a motivational and DT-based intervention that can be delivered on a mobile platform. In the United States almost $95 \%$ of adults own a mobile phone [23]. Text message interventions have demonstrated efficacy for health promotion and behavior change, with personalized messages showing the greatest benefit [24]. These platforms also offer the advantage of being delivered outside of structured treatment sessions and allow for the content, timing, and frequency of messages to be individually tailored to times when certain skills and motivational reminders are most salient [25].

This paper describes the study design and procedures for a behavioral treatment development trial that examines the feasibility, acceptability, and preliminary efficacy of a digital health intervention (iCOPE) designed to promote engagement in and adherence to buprenorphine through motivational enhancement and DT skills training among individuals initiating outpatient addiction treatment.

\section{Methods}

\section{Study design overview}

The current study meets the objectives of Stage I of the NIDA Behavioral Therapies Development Program, which provides a conceptual framework grounded in basic science, for intervention development [26]. Stage I includes two phases-Stage 1A and Stage 1B. Stage $1 \mathrm{~A}$ involves the creation of a new intervention, while Stage 1B establishes feasibility through pilot testing [26]. In Phase 1 (Stage 1A), our team will develop and refine, through formative evaluation, an interactive computer- and text message-delivered PFI that incorporates DT skills training for persons who have elected to initiate outpatient buprenorphine (iCOPE). Specifically, this 
intervention is designed to promote medication induction and stabilization following standard clinic intake procedures. All participants will receive standard outpatient buprenorphine treatment, including medication visits, counseling, case management, and peer recovery (treatment as usual; TAU). In Phase 2 (Stage 1B), we will conduct a pilot randomized controlled trial. Eighty patients with a history of OUD, actively seeking outpatient buprenorphine, will be randomly assigned to iCOPE or TAU, using a 3:1 randomization ratio. We propose a $3: 1$ randomization ratio to maximize the information gained about the iCOPE intervention while including a comparison condition. All participants will complete assessments at treatment initiation as well as 1-, 4-, 8and 12-weeks post-initiation. These data will be used to inform the development of a large-scale, fully-powered trial. At the time of this report, recruitment for Stage 1A is ongoing.

\section{Stage $1 \mathrm{~A}$}

Qualitative, in-depth, individual interviews are currently being conducted with patients actively engaged in buprenorphine treatment (target $N=24$ ). The sample is balanced by gender, primary type of opioid use (prescription pills; heroin), and phase of recovery [early ( $\leq 8$ weeks of treatment) vs. late $(>8$ weeks of treatment)]. Interviews will continue until saturation is achieved. Following informed consent, demographic and clinical data are obtained. Interviews are conducted by the Principal Investigator (KJL) or a trained Research Assistant, and follow a semi-structured interview protocol that stems from primary research questions and project goals. Specific questions focus on participants' use of computers, mobile phones, and other technologies; prior engagement in MOUD; barriers/facilitators to engaging in MOUD; reactions to and perceived usefulness of proposed intervention; and preferences, benefits, and likelihood of engaging in digital health interventions. All interviews are conducted in a private office to ensure confidentiality and are digitally recorded. Each interview takes approximately 60-90 min to complete; participants receive a $\$ 30$ gift card for their time/effort. Interviews are transcribed by a professional agency, and the written transcripts are later reviewed to resolve discrepancies.

Qualitative analyses will be conducted to inform intervention development and refinement. Both thematic (deductive) and data driven (inductive) codes will be utilized. Deductive codes will be drawn from the topics in questions used to facilitate the interviews; inductive codes will capture additional concepts that emerge from the participants. Early interviews will be coded by three team members, until stability of the coding structure is reached. All interviews thereafter will be independently coded by two team members using the coding scheme, then compared to ensure agreement. Agreed upon codes will be entered into NVivo. Throughout the process, a framework matrix will be created. This data reduction tool, a matrix of cases and themes based on interview debriefs and individual interview codes, will be used to track emergent ideas and concepts that impact intervention design and future interviews [27-29]. After every few interviews, research team members will examine the framework matrix, identify reoccurring major themes, make changes to intervention content as appropriate, then test the edits in subsequent interviews. This method will allow for quick, iterative turnaround of participant feedback to intervention edits and modifications of interview questions.

We will further refine the intervention by recruiting an additional 16 participants for open pilot testing. These participants will be in the initial 8 weeks of treatment and will be balanced by gender and primary type of opioid use. At the end of the iCOPE intervention, semi-structured interviews, in combination with measures of protocol adherence, will be used to evaluate and then improve upon the intervention's appropriateness and comprehensibility. During the interviews, participants will be queried about their comfort with technology (computer and text message); ease of using the technology formats; level of understanding of intervention content; perceived usefulness of material; satisfaction with format and content; likelihood of recommending the intervention; likelihood of continued use; and suggestions for improvement. To guide refinements, we will collect data on the feasibility and acceptability of the intervention. Feasibility will be determined by assessing study recruitment and refusal rates, intervention completion, follow-up rates, and rates of study attrition. Acceptability will be determined by the (a) System Usability Scale, a participant-completed, reliable and valid metric for measuring usability and acceptability of technologies; [30-32] and (b) Relative Subjective Count, the quotient of the participant's estimate of the number of times the system delivers a text, divided by the number of texts actually delivered [33]. All proposed changes will be integrated into the final version of $\mathrm{iCOPE}$ for testing in Phase 2.

\section{Stage 1B}

Stage $1 \mathrm{~B}$ will employ a randomized controlled trial design to pilot test iCOPE relative to the TAU control condition (see description of treatment conditions below). Adult patients $(N=80)$ meeting eligibility criteria will be randomly assigned to either the iCOPE or TAU conditions.

Following the initial evaluation, in which patients are admitted to buprenorphine treatment, staff will refer potentially eligible patients to study personnel. Interested 
participants will meet with study personnel in a private setting at the clinic. Informed consent will be obtained, and final eligibility will be determined. Eligible participants will complete a baseline assessment, including two brief behavioral measures of DT (Mirror Tracing and Breath Holding; see Assessments). Participants will then be randomly assigned to iCOPE or TAU. Participants in the iCOPE condition will complete the brief computerized intervention. They will also be informed about the text message element of the intervention and instructed in these procedures. We will recruit 80 participants who will be assigned to condition at a 3:1 ratio, with 60 participants assigned to iCOPE and 20 participants assigned to TAU. Participants will be scheduled to return to the clinic for follow-up appointments consisting of an online survey, behavioral DT measures, and urine collection. Follow-ups will occur at 1-, 4-, 8- and 12-weeks posttreatment initiation.

\section{Participants}

Participants will include 80 males and females between the ages of 18 and 75 initiating OUD treatment, specifically the use of buprenorphine. For inclusion, participants must meet current Diagnostic and Statistical Manual of Mental Disorders, 5th Edition (DSM-5), criteria for OUD and have access to a cell phone with text message capability. To reduce the risk of adverse events and potential confounds, we will employ the following exclusion criteria: (1) active suicidality and/or psychosis that would interfere with the ability to participate in the intervention, (2) not fluent in English as translation is beyond the current study resources, and (3) limited mental capacity or inability to provide informed written consent.

\section{Study setting}

The Lifespan Recovery Center at Rhode Island Hospital will serve as the proposed recruitment site. The Lifespan Recovery Center is an outpatient addiction treatment program that offers buprenorphine and ancillary services (e.g., counseling, case management, peer recovery). The clinical team includes: four physicians, four therapists, a case manager, and a peer recovery specialist. On average, there are approximately 20 new patient admissions per month. As part of routine clinical care at the Lifespan Recovery Center, patients typically attend an intake evaluation and medication induction appointment, followed by weekly follow-up visits for the initial 8 weeks of treatment (stabilization). Once stabilized, patients continue to attend monthly follow-up appointments for the duration of treatment.

\section{Compensation and retention}

In an effort to bolster study retention, we have aligned study follow-up assessments with routine clinic visits. Additionally, follow-up reminders will occur via emails, telephone calls, and text messages. Participants will earn a \$25 gift card for completing the initial assessment and a \$20 gift card for completing each follow-up assessment. Participants completing all required follow-ups will earn an additional \$25 gift card. Participants have the potential to earn up to $\$ 130$ for completing all study assessments.

\section{Intervention conditions iCOPE}

iCOPE will involve two delivery modes: computer and text message (see Fig. 1). The proposed intervention will initially target motivational processes in combination with introductory strategies for managing physical and emotional distress through a single, brief, computerdelivered, session followed by 8 weeks of theoreticallyinformed text messages intended to enhance motivation and promote DT (see Table 1).

The initial intervention phase will be delivered via computer following routine intake procedures at the recruitment site. We elected to have participants complete the computer-based intervention on-site, instead of allowing participants to complete independently on their own device, to optimize fidelity of the intervention given that it is a Stage 1 trial. We will evaluate participant preference for computer delivery in the open pilot trial to determine if expanding the intervention to home devices is warranted in the future. iCOPE will be presented in a way that encourages participants to be mindful of their behaviors in a non-confrontational manner. The intervention has two aims: (a) engage patients in a decisional balance exercise designed to evaluate the perceived advantages/disadvantages of making a behavior change (abstinence from opioids, adhering to buprenorphine) to enhance motivation and (b) provide concrete strategies to better tolerate emotional and physical discomfort to persist with identified behavioral goals while initiating treatment. First, participants will be guided through a decisional balance exercise and queried about perceived barriers to treatment. Next, this information will be summarized to deliver feedback about personal motivators and recommended DT coping skills.

The second intervention phase will be delivered via text message and will focus on (a) promoting motivation for abstinence over and above the reinforcing effects of opioids and (b) emphasizing adaptive strategies for tolerating physical and psychological discomfort. To do this, text messages will be strategically delivered during the 


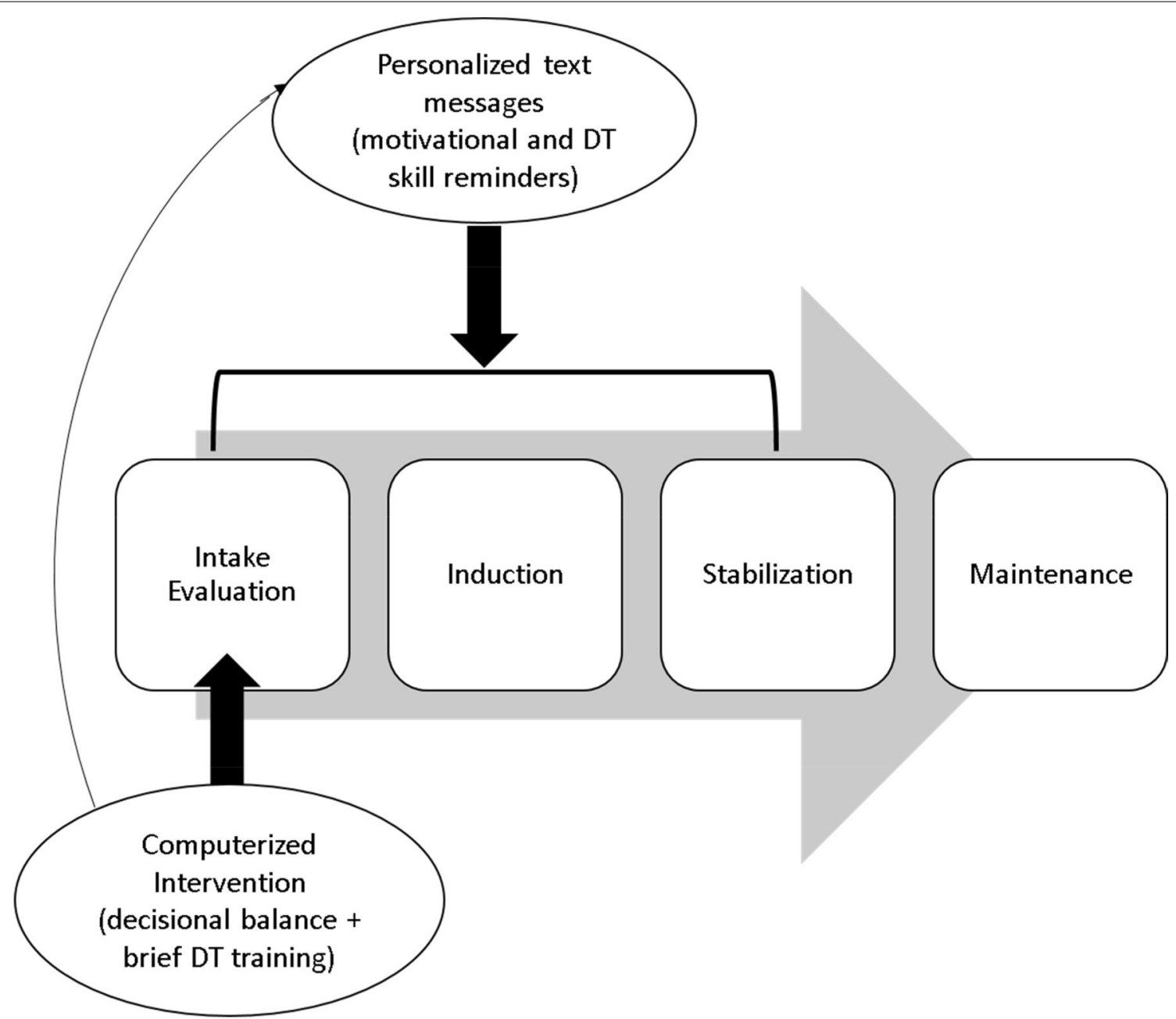

Fig. 1 Overview of Proposed iCOPE Intervention

Table 1 Description of iCOPE treatment content

Intervention Computer intervention components type

PFI Evaluate advantages and disadvantages of behavior change

Elicit recognition of problem and motivating factors for change

Assist with setting personal change goals for opioid use and treatment

DT Acceptance: education on meaning of acceptance; strategies to promote acceptance of reality

Self-soothing: education on using five senses to comfort self during times of distress

Distraction: education on using distraction skills to change emotional response to distressing stimuli

Improving the moment: education on methods to replace immediate negative events/states with more positive ones
Text message intervention components

Reminders of the advantages of behavior change elicited during initial intervention

Reminders of personal motivators

Reminders of personal goals

Acceptance: encourage acceptance-based strategies to use in moments of distress

Self-soothing: suggestions of ways to comfort self during times of distress

Distraction: reminders of distraction strategies to change emotional responding

Improving the moment: encourage methods to replace negative with positive states 
initial 8 weeks of stabilization which are considered a "high-risk" period (see Fig. 1). The text messages will be personalized in nature and offer skills training (reminders of previously learned DT skills) and motivational messages (content directly derived from decisional balance exercise; see Table 1). The frequency and timing of text messages will be determined through prior formative work; however, we anticipate sending at least two automated text messages each day. A variety of messages will be delivered throughout the eight-week period and will create (a) a natural progression in skill-building, (b) continued motivation for engagement, and (c) responsiveness to participants' needs through personalization. We will create a variety of text messages that provide concrete DT skills as well as personalized messages reminding participants of their identified goals and personal motivators (e.g., health, family, finances). Further, we anticipate providing participant-driven text messages to provide tailored support in the moments needed. For example, if a participant texts "CRAVING" or "STRESS" to the automated program, indicating the need for additional support on that topic, the participant would receive a relevant skills-based message. Messages will be delivered to participants' personal cell phones using an automated computerized system based upon pre-specified delivery algorithms [34, 35].

\section{Treatment as usual (TAU)}

The TAU condition will consist of comprehensive outpatient buprenorphine treatment, including medication management, counseling, case management, and peer recovery. TAU was selected as the comparison condition as it represents a robust and evidence-based approach to the treatment of OUD. The goal of this phase of treatment development is to evaluate the initial feasibility, acceptability and preliminary efficacy of the proposed intervention through a small pilot trial. Decisional frameworks used to select an appropriate control condition for randomized controlled trials for behavioral interventions suggest that at earlier stages of treatment development it may be favorable to select a TAU condition. Inclusion of a rigid control condition (e.g., health education condition) could result in the underestimation of the effect size and yield null findings in a trial with insufficient statistical power to detect effects [36]. TAU is currently the gold standard of care for treating this population, and therefore, an adequate control condition for this stage of development and evaluation.

\section{Assessments}

\section{Diagnostic and screening assessment}

During routine clinic procedures, patients are evaluated for the presence of DSM-5 OUD, active suicidality and/or psychosis, and interest in initiating buprenorphine through a shared decision-making process. A Demographic-Treatment History Questionnaire will assess gender, age, race/ethnicity, education, occupation, income, substance use treatment history, and access to a cell phone with text message capability (see Table 2).

\section{Primary outcome}

The primary outcome of this study is percentage of days positive for drug use in between assessments (self-report and biochemically verified; see Table 2). The interviewerdelivered Timeline Follow-Back will assess daily opioid use, other illicit substances (e.g., cocaine), and alcohol

Table 2 Overview of timing of measurement

\begin{tabular}{|c|c|c|c|c|c|}
\hline Measure & Intake & 1-Week & 4- Weeks & 8-Weeks & 12- Weeks \\
\hline Demographics-treatment history & $x$ & & & & \\
\hline Distress tolerance scale & $X$ & $x$ & $x$ & $x$ & $x$ \\
\hline Mirror-tracing persistence task & $X$ & $x$ & $x$ & $x$ & $x$ \\
\hline Breath-holding task & $X$ & $x$ & $x$ & $x$ & $x$ \\
\hline Difficulties with emotional regulation scale & $x$ & $x$ & $x$ & $x$ & $x$ \\
\hline UPPS-P impulsive behavior scale & $X$ & $x$ & $x$ & $x$ & $x$ \\
\hline Positive affect negative affect schedule & $x$ & $x$ & $x$ & $x$ & $x$ \\
\hline Decisional balance scale & $x$ & & & & \\
\hline Timeline follow-Back & $x$ & $x$ & $x$ & $x$ & $x$ \\
\hline Urine toxicology screens & $x$ & $x$ & $x$ & $x$ & $x$ \\
\hline Readiness ruler & $x$ & $x$ & $x$ & $x$ & $x$ \\
\hline Client satisfaction questionnaire & $x$ & & & $x$ & \\
\hline System usability scale & & & & $x$ & \\
\hline Relative subjective count & & & & $x$ & \\
\hline
\end{tabular}


use. It has demonstrated good reliability [37] and has been validated for the assessment of opioids and other substance use [38]. Urine Toxicology Screens will be administered at all time points to screen for the presence of opioids and other illicit substances.

\section{Secondary outcomes}

This study will also evaluate other clinically-relevant outcomes, including: percentage of days positive for buprenorphine administration in between assessments (self-report via Timeline Follow-Back and biochemically verified via Urine Toxicology Screens) and retention in treatment (see Table 2). Retention will be determined by the total number of clinic visits attended, and treatment discontinuation based on electronic medical record review. This study also seeks to identify potential mechanisms that may underlie the efficacy of iCOPE. Therefore, we have selected several other secondary outcomes of interest. These include motivation, distress tolerance, emotional and behavioral self-regulation skills, and negative affect (see Table 2). The readiness ruler assesses current level of motivation to change opioid use [39]. Self-reported DT will be assessed by the Distress tolerance scale [40], which evaluates general tolerance of psychological distress. Behavioral assessments of DT will include the computerized mirror-tracing persistence task [41] to assess psychological DT and a breath-holding task to assess physical DT. The positive affect and negative affect schedule will be used to evaluate negative affect throughout the study [42]. The difficulties with emotion regulation scale [43] will be used to assess the degree to which respondents experience dysregulated emotional states (emotional self-regulation). The UPPS-S impulsive behavior scale will assess a 5 -factor model of impulsivity (behavioral self-regulation) [44].

\section{Feasibility and acceptability}

Treatment satisfaction will be assessed by the client satisfaction questionnaire-8 [45] following the computerized intervention delivered at intake and following the text message portion of the study. Usability and acceptability of the text message intervention will be evaluated by the system usability scale [30-32] while satisfaction will be assessed through the relative subjective count (rsc). Low RSC correlates with increased patient satisfaction; high RSC reflects poor acceptability of a technology [33] see Table 2.

\section{Data analysis \\ Overview}

Considering this is a pilot study, analyses will have the goal of establishing feasibility, acceptability, and estimation of effect sizes, with modest expectations for rejection of null hypotheses. As a first step, the equivalence of treatment conditions regarding key baseline variables will be assessed. This will involve comparisons of treatment conditions on demographics and baseline levels of potential treatment moderators (e.g., gender and age). Should conditions differ on any characteristic, these variables will be placed in models as interactions with group assignment, along with its main effect, and also in a distinct model with the interaction removed. The model with the lowest Akaike Information Criterion (AIC) will be retained. Other preliminary analyses will include patterns of missing data, dropout rates, distributional properties of dependent and other measures, and correlations among outcome measures. Following the intent-to-treat principle, all randomized participants will be included in the analyses. Given the developmental nature of this study, our primary goal is to yield a stable estimate of the effect size rather than to find statistically significant differences. We are aware of the dangers of relying exclusively on small pilot studies to gauge the promise of novel interventions [46]. These effect size estimates have a large standard error, and we primarily will be examining a pattern of results that is supportive of iCOPE, at which point a full-scale trial will be designed to test for a clinically meaningful effect size. Group means on continuous variables typically begin to stabilize around 15 participants per group. For dichotomous or categorical variables, a larger sample size may be needed to provide stable odds ratios for effect size estimates. Our proposed sample size of 60 participants in $\mathrm{iCOPE}$ and 20 participants in HE will allow us to evaluate the potential of iCOPE to improve treatment outcomes while maximizing the number of participants in the iCOPE condition to fully assess the feasibility and acceptability of the intervention. A formal power analysis was not completed as this is a pilot trial, which is not designed to detect statistically significant differences between intervention groups [55].

\section{Primary pilot trial analyses}

Tests of the effects of treatment on the primary outcome variables (abstinence, adherence, retention) will be conducted using a fractional logit model [47] estimated by Generalized Estimating Equations (GEE) [48, 49]. GEE is a quasi-likelihood estimation method of repeated measures for modeling of covariance structures when outcomes are correlated across time. It allows for inclusion of both categorical and continuous independent variables. The fractional logit model can be used for any fractional outcome with a range of 0 to 1 and can be readily implemented using SAS PROC GLIMMIX. An advantage of GEE over ANOVA is that GEE models address nesting by adjusting the standard errors of the test statistics based on the covariances (and variances) of nested 
observations, rather than depending on calculating differences. These variances and covariances can be modeled based on all data available. The primary, between groups, independent variable in the above GEE is treatment group. Variables measured at baseline will be examined using screening runs prior to primary analyses to see which of these baseline variables are most strongly associated with the outcomes. Those that show significant relations with outcome will be entered as additional covariates in the primary analyses unless there are concerns of multicollinearity. The linear effect of time also will be included as a covariate in these analyses. We will test for non-linear (i.e., linear plus quadratic) effects of time for the repeated measures. Analyses will be conducted separately on two overlapping samples. Following the intent-to-treat principle, all randomized participants will be included in the first set of analyses. This is the most conservative approach and represents our main outcome analysis. We also will conduct analyses on subjects who completed the computer-based intervention, the "as-treated" analysis. Although subject to more bias, especially if dropout is a problem, this latter approach answers more directly the question of intervention efficacy by providing an estimate of the maximal effects attained by an intervention. Similar results with both approaches increases confidence in the findings.

\section{Exploratory analyses}

Potential treatment mechanisms (motivation, distress tolerance, emotional and behavioral self-regulation skills, and negative affect) will be examined separately, rather than simultaneously, given our limited sample size and ability to tease apart the relative contributions of related variables. Mediation analyses will be temporally ordered, such that mediators are assessed after the intervention (controlling for baseline levels of mediators), and outcomes will be assessed at a later time point than the mediators (controlling for baseline levels of outcomes). Though power will be limited, these analyses will at least inform us whether the hypothesized mediators are (a) likely to be influenced by treatment and (b) are likely to associate with our primary outcomes. Conducting these analyses will also provide information to plan a future, larger-scale trial.

\section{Discussion}

The prevalence of OUD has reached epidemic rates in United States, and opioid-involved overdoses are now the country's leading cause of injury deaths [50]. MOUD, including the use of buprenorphine, is effective at producing significant reductions in illicit opioid use as well as improvements in health-related outcomes (e.g., reduced risk of HIV) $[6,51]$. However, despite the many advantages associated with buprenorphine treatment, nearly half of participants are unable to achieve stabilization [7], and many patients lapse or discontinue treatment in the initial weeks [8]. This is notable as early lapses are related to poor treatment prognosis and retention [8, 52]. Given high rates of noncompliance and/ or discontinuation, there have been recent calls to find innovative interventions to enhance motivation, adherence, and retention in buprenorphine treatment [53].

Consistent with a negative reinforcement model of substance use, aversive internal states, that occur in the context of early periods of abstinence, may contribute to difficulties with buprenorphine stabilization [54]. Accordingly, an intervention that (a) cultivates motivation for abstinence over and above the reinforcing effects of opioids and (b) teaches adaptive strategies for tolerating physical and psychological discomfort may optimize stabilization on buprenorphine to improve the likelihood of long-term recovery. This Stage 1 behavioral treatment development trial seeks to develop, and pilot test, an innovative digital health intervention that aims to enhance motivation to engage in treatment and increase tolerance of distress to facilitate buprenorphine stabilization by providing skills training and motivational reminders in 'real-time.'

At the conclusion of this study, our goals are to (1) evaluate the feasibility, acceptability, and preliminary efficacy of the iCOPE intervention components and (2) explore potential mechanisms underlying the effects of the intervention. Results of this study will be used to determine whether to proceed with further testing through a large-scale, fully-powered trial. Even if this study produces null effects of the proposed intervention, the data will still yield valuable information to inform future intervention development efforts.

This work has the potential to improve treatment outcomes by reducing illicit opioid use, increasing adherence/retention, and preventing future incidence of overdose. If successful, this study has high clinical and public health significance by developing and pilot testing the preliminary efficacy of an intervention that may reach a high-risk and vulnerable segment of the population. Further, by leveraging digital health platforms, this proposal has the potential for high scalability and impact.

\footnotetext{
Abbreviations

OUD: Opioid use disorder; MOUD: Medications for opioid use disorder; DT: Distress tolerance; PFI: Personalized feedback intervention; TAU: Treatmentas-usual; DSM-5: Diagnostic and statistical manual of mental disorders, 5th Edition; RSC: Relative subjective count; AIC: Akaike information criterion; GEE: Generalized estimating equations.
}

Acknowledgements Not applicable. 


\section{Authors' contributions}

KJL is the Principal Investigator for the study, led the conceptualization of the study and the drafting of the original grant proposal, and developed a first draft of the manuscript. SR, KC, MLR and JR are mentors, and CS is the research assistant of the study. SR, KC, MLR and JR also contributed to the writing of the original grant proposal and successive drafts of the manuscript. CS contributed to successive drafts of the manuscript. All authors read and approved the final manuscript.

\section{Funding}

Research reported in this publication was supported by the National Institute on Drug Abuse of the National Institutes of Health under Award Number K23DA046482 awarded to Dr. Langdon. Drs. Rich, Ramsey, Ranney and Langdon are all supported, in part by a National Institute of General Medical Studies Grant (P20 GM125507). The content is solely the responsibility of the authors and does not necessarily represent the official views of the National Institutes of Health.

\section{Availability of data and materials}

Not applicable.

\section{Ethics approval and consent to participate}

The work described has been carried out in accordance with The Code of Ethics of the World Medical Association (Declaration of Helsinki) for experiments involving human subjects, and the procedures have been approved by the Lifespan Institutional Review Board.

\section{Consent for publication}

Not applicable.

\section{Competing interests}

The authors declare that they have no competing interests.

\section{Author details}

1 Department of Psychiatry, Rhode Island Hospital, 146 West River Street, Suite 11A, Providence, RI 02904, USA. ${ }^{2}$ Department of Psychiatry and Human Behavior, Alpert Medical School of Brown University, Providence, USA. ${ }^{3}$ Department of Medicine, Alpert Medical School of Brown University, Providence, USA.

${ }^{4}$ Division of General Internal Medicine, Department of Medicine, Rhode Island Hospital, Providence, USA. ${ }^{5}$ Department of Emergency Medicine, Alpert Medical School Brown University, Providence, USA. ${ }^{6}$ Department of Behavioral and Social Sciences, Brown University School of Public Health, Providence, USA. ${ }^{7}$ Brown University School of Public Health, Center for Alcohol and Addiction Studies, Providence, USA. ${ }^{8}$ Emergency Digital Health Innovation Program, Brown University, Providence, USA. ${ }^{9}$ Department of Epidemiology, Brown University School of Public Health, Providence, USA.

Received: 26 November 2019 Accepted: 17 April 2020

Published online: 29 April 2020

\section{References}

1. Scholl L, Seth P, Kariisa M, Wilson N, Baldwin G. Drug and opioid-involved overdose deaths-United States, 2013-2017. Morb Mortal Wkly Rep. 2018;67:1419-27.

2. 2017 National Survey on Drug Use and Health, Mortality in the United States; 2016.

3. National Academies of Sciences, Engineering, and Medicine; 2019.

4. Boothby LA, Doering PL. Buprenorphine for the treatment of opioid dependence. Am J Health Syst Pharm. 2007;64:266-72.

5. Medication-Assisted Treatment Buprenorphine. In: Substance Abuse and Mental Health Services Administration. 2016. https://www.samhsa.gov/ medication-assisted-treatment/treatment/buprenorphine. Accessed 11 Nov 2019

6. Thomas CP, Fullerton CA, Kim M, Montejano L, Lyman DR, Dougherty RH, et al. Medication-assisted treatment with buprenorphine: assessing the evidence. Psychiatric Serv. 2014:65:158-70.
7. Hser YI, Saxon AJ, Huang D, Hasson A, Thomas C, Hillhouse M, et al. Treatment retention among patients randomized to buprenorphine/naloxone compared to methadone in a multi-site trial. Addiction. 2014;109:79-87.

8. Stein MD, Herman DS, Kettavong M, Cioe PA, Friedmann PD, Tellioglu $\mathrm{T}$, et al. Antidepressant treatment does not improve buprenorphine retention among opioid-dependent persons. J Subst Abuse Treat. 2010;39:157-66.

9. Novak A, Burgess ES, Clark M, Zvolensky MJ, Brown RA. Anxiety sensitivity, self-reported motives for alcohol and nicotine use, and level of consumption. J Anxiety Disord. 2003;17:165-80.

10. McHugh RK, Kneeland ET. Affective vulnerability in substance use disorders. Curr Opin Psychol. 2019;30:54-8.

11. Brown RA, Lejuez CW, Kahler CW, Strong DR. Distress tolerance and duration of past smoking cessation attempts. J Abnorm Psychol. 2002;111:180-5.

12. Lejuez CW, Paulson A, Daughters SB, Bornovalova MA, Zvolensky MJ. The association between heroin use and anxiety sensitivity among inner-city individuals in residential drug use treatment. Behav Res Ther. 2006;44:667-77.

13. Trafton JA, Gifford EV. Behavioral reactivity and addiction: the adaptation of behavioral response to reward opportunities. J Neuropsychiatry Clin Neurosci. 2008;20:23-35.

14. McHugh RK, Otto MW. Profiles of distress intolerance in a substancedependent sample. Am J Drug Alcohol Abuse. 2012;38:161-5.

15. McHugh RK, Weiss RD, Cornelius M, Martel MO, Jamison RN, Edwards RR. Distress intolerance and prescription opioid misuse among patients with chronic pain. J Pain. 2016;17:806-14.

16. Daughters SB, Lejuez CW, Bornovalova MA, Kahler CW, Strong DR, Brown RA. Distress tolerance as a predictor of early treatment dropout in a residential substance abuse treatment facility. J Abnormal Psychol. 2005; 114(4):729.

17. Strong DR, Brown RA, Sims M, Herman DS, Anderson BJ, Stein MD. Persistence during stress-challenge predicts lapse to opiate use during buprenorphine treatment. J Addiction Med. 2012;6:19-25.

18. Daughters SB, Lejuez CW, Kahler CW, Strong DR, Brown RA. Psychological distress tolerance and duration of most recent abstinence attempt among residential treatment-seeking substance abusers. Psychol Addictive Behaviors. 2005;19(2):208.

19. Bornovalova MA, Gratz KL, Daughters SB, Hunt ED, Lejuez CW. Initial $\mathrm{RCT}$ of a distress tolerance treatment for individuals with substance use disorders. Drug Alcohol Depend. 2012;122:70-6.

20. Janis IL, Mann L. Decision-making: a psychological analysis of conflict, choice, and commitment. New York: The Free Press; 1997.

21. Collins SE, Kirouac M, Lewis MA, Witkiewitz K, Carey KB. Randomized controlled trial of web-based decisional balance feedback and personalized normative feedback for college drinkers. J studies Alcohol Drugs. 2014;75(6):982-92

22. Lee CM, Neighbors C, Kilmer JR, Larimer ME. A brief, web-based personalized feedback selective intervention for college student marijuana use: a randomized clinical trial. Psychol Addict Behav. 2010;24:265-73.

23. http://www.pewinternet.org/fact-sheet/mobile/; Accessed Jan 12, 2017.

24. Head KJ, Noar SM, lannarino NT, Harrington NG. Efficacy of text messagebased interventions for health promotion: a meta-analysis. Soc Sci Med. 2013;97:41-8

25. Muench F, Weiss RA, Kuerbis A, Morgenstern J. Developing a theory driven text messaging intervention for addiction care with user driven content. Psychol Addictive Behavior. 2013;27:315-21.

26. Onken LS, Carroll KM, Shoham V, Cuthbert BN, Riddle M. Re-envisioning clinical science: unifying the discipline to improve the public health. Clin Psychol Sci. 2014;2(1):22-34.

27. Ritchie J, et al. Qualitative research practice: a guide for social science students and researchers. Tucson: Sage Publishing; 2013.

28. Gale NK, et al. Using the framework method for the analysis of qualitative data in multidisciplinary health research. BMC Med Res Methodol. 2013;13:117.

29. Smith J, Firth J. Qualitative data analysis: the framework approach. Nurse Res. 2011;18:52-62.

30. Brooker J. Digital Equipment Co Ltd. United Kingdom: Reading; 1986.

31. Bevan N, Kirakowski J, Maissel J. What is Usability? In: Bullinger H-J, editor. Human aspects in computing: design and use of interactive systems and work with terminals. Amsterdam: Elsevier; 1991. 
32. Kirakowski J, Corbett M. Measuring User Satisfaction. In: Jones DM, Winder R, editors. People and Computers IV. Cambridge: Cambridge University Press; 1988

33. Picard RW, Liu KK. Relative subject count and assessment of interruptive technologies applied to mobile monitoring of stress. Int J Hum Comput Stud. 2007;65:361-75

34. Ranney ML, Pittman SK, Dunsiger S, Guthrie KM, Spirito A, Boyer EW, Cunningham RM. Emergency department text messaging for adolescent violence and depression prevention: a pilot randomized controlled trial. Psychol Serv. 2018;15(4):419.

35. Ranney ML, Freeman JR, Connell G, Spirito A, Boyer E, Walton M, Guthrie KM, Cunningham RM. A depression prevention intervention for adolescents in the emergency department. J Adolescent Health. 2016;59:401-10

36. Gold SM. Control conditions for randomised trials of behavioural interventions in psychiatry: a decision framework. Lancet Psychiatry. 2017;4(9):725-32.

37. Robinson SM, Sobell LC, Sobell MB, Leo Gl. Reliability of the timeline followback for cocaine, cannabis, and cigarette use. Psychol Addict Behav. 2014:28:154-62.

38. Hjorthøj CR, Hjorthøj AR, Nordentoft M. Validity of timeline follow-back for self-reported use of cannabis and other illicit substances-systematic review and meta-analysis. Addictive Behaiors. 2012;37:225-33.

39. Hesse M. The Readiness Ruler as a measure of readiness to change polydrug use in drug abusers. Harm Reduction J. 2006;3:3.

40. Simons JS, Gaher RM. The distress tolerance scale: development and validation of a self-report measure. Motivation Emotion. 2005;29:83-102.

41. Strong DR, Lejuez CW, Daughters SB, Marinello M., Kahler CW, Brown RA. The computerized mirror tracing task version 1: Unpublished manual; 2003

42. Watson D, Clark LA, Tellegen A. Development and validation of brief measures of positive and negative affect: the PANAS scales. J Pers Soc Psychol. 1988;54:1063-70.

43. Gratz KL, Roemer L. Multidimensional assessment of emotion regulation and dysregulation: development, factor structure, and initial validation of the difficulties in emotion regulation scale. J Psychopathol Behav Assess. 2004;36:41-54.

44. Whiteside SP, Lynam DR. The five factor model and impulsivity: using a structural model of personality to understand impulsivity. Personality Individ Differ. 2001;30:669-89.
45. Larsen DL, Attkisson C, Hargreaves WA, Nguyen TD. Assessment of client/ patient satisfaction: development of a general scale. Evalu Program Plan. 1979:2:197-207.

46. Kraemer HC, Mintz J, Noda A, Tinklenberg J, Yesavage JA. Caution regarding the use of pilot studies to guide power calculations for study proposals. Arch Gen Psychiatry. 2006;63:484-9.

47. Papke LE, Wooldridge JM. Panel data methods for fractional response variables with an application to test pass rates. J Econ. 2008;145:121-33.

48. Hall SM, Delucchi KL, Velicer WF, Kahler CW, Ranger-Moore J, Hedeker D, Tsoh JY, Niaura R. Statistical analysis of randomized trials in tobacco treatment: longitudinal designs with dichotomous outcome. Nicotine Tob Res. 2001;3:193-202.

49. Llang K-Y, Zeger SL. Longitudinal data analysis using generalized linear models. Biometrika. 1986:73:13-22.

50. Centers for Disease Control. Increases in drug and opioid overdose deaths United States, 2000-2014. Morb Mortal Wkly Rep. 2016;64:1378-82

51. Connock M, Juarez-Garcia A, Jowett S, Frew E, Liu Z, Taylor RJ, et al. Methadone and buprenorphine for the management of opioid dependence: a systematic review and economic evaluation. Health Technol Assess. 2007;11:1-171.

52. Stein MD, Cioe PA, Friedmann PD. Buprenorphine retention in primary care. J Gen Intern Med. 2005;20:1038-41.

53. Carroll KM, Weiss RD. The role of behavioral interventions in buprenorphine maintenance treatment: a review. Am J Psychiatry. 2017;174:738-47

54. Brown RA, Bloom EL, Hecht J, Moitra E, Herman DS, Stein MD. A pilot study of stress tolerance treatment for opiate dependent patients initiating buprenorphine: rationale, methodology, and outcomes. Behavior Modif. 2016:38:730-59.

55. Leon AC, Davis LL, Kraemer HC. The role and interpretation of pilot studies in clinical research. J Psychiatr Res. 2011;45:626-9.

\section{Publisher's Note}

Springer Nature remains neutral with regard to jurisdictional claims in published maps and institutional affiliations.
Ready to submit your research? Choose BMC and benefit from:

- fast, convenient online submission

- thorough peer review by experienced researchers in your field

- rapid publication on acceptance

- support for research data, including large and complex data types

- gold Open Access which fosters wider collaboration and increased citations

- maximum visibility for your research: over 100M website views per year

At $\mathrm{BMC}$, research is always in progress.

Learn more biomedcentral.com/submissions 